\title{
Comprehensive Understanding of Carrier Mobility in MOSFETs with Oxynitrides and Ultrathin Gate Oxides
}

\author{
T. Ishihara*, J. Koga*, and S. Takagi** \\ * Advanced LSI Technology Laboratory, Corporate Research \& Development Center, \\ Toshiba Corporation, 8 Shinsugita-cho, Yokohama 235-8522, Japan \\ ishihara@amc.toshiba.co.jp \\ **Department of Frontier Informatics, Graduate School of Frontier Science, The \\ University of Tokyo, 7-3-1 Hongo, Bunkyo-ku, Tokyo, 113-8656, Japan
}

\begin{abstract}
Inversion-layer mobility in MOSFETs is quantitatively examined by taking MOSFETs with oxynitride and ultra-thin gate oxides as examples. It was shown that additional Coulomb scattering due to charged impurities in poly-Si gate and due to fixed charges in oxynitride gate oxides is responsible for the mobility degradation in low effective electric field region for ultra-thin gate oxides and oxynitride gate oxides, respectively. For high effective electric field region, the different behavior of inversion-layer mobility between electrons and holes associated with oxynitridation can be reasonably well explained by the appropriate choice of the form of the roughness correlation function. This paper focuses on the fact that Coulomb and surface roughness scattering are key scattering components for the unified understanding of carrier mobility in inversion layers of future MOSFETs.
\end{abstract}

\section{Introduction}

Thinner gate oxide layer, high-k dielectric layer, or oxynitride gate layer are considered to be promising candidates for application as gate dielectrics in future MOSFETs characterized by aggressive scaling down of device sizes. The significant change in inversion-layer mobility associated with these gate dielectrics has been observed experimentally $[1,2,3,4]$. For example, in MOSFETs with oxynitride gate oxides low field mobility shows significant degradation for both electrons and holes, while high field mobility exhibits different behavior for electrons and holes, as is shown in Fig. 1 [1]. In MOSFETs with ultra-thin gate oxides, the inversion-layer mobility exhibits a significant reduction in gate oxides thinner than a critical thickness [3,4] (Fig. 2). Although much theoretical work has been done on inversion-layer mobility in MOSFETs with pure and thicker gate oxides, the origin of the change in inversion-layer mobility in MOSFETs with the above-mentioned gate dielectrics has not been fully understood yet. Considering the fact that the dominant scattering components in low and high field region are Coulomb and surface roughness scattering, respectively, Coulomb and surface roughness scattering are key scattering components for the comprehensive understanding of transport properties in inversion 
layers of future MOSFETs [5]. By taking MOSFETs with oxynitride and ultra-thin gate oxides as examples, we examine transport properties in inversion layers based on the model calculation of the mobility limited by Coulomb and surface roughness scattering. Since the physical basis of the models used is important, we describe in section 2 the Coulomb and surface roughness scattering model used in this study. By comparing the calculated and the experimental results of the inversion-layer mobility, we show in sections 3 and 4 that the change in the inversion-layer mobility can be comprehensively understood by considering additional Coulomb scattering and modulated surface roughness scattering. The conclusions are given in section 5 .
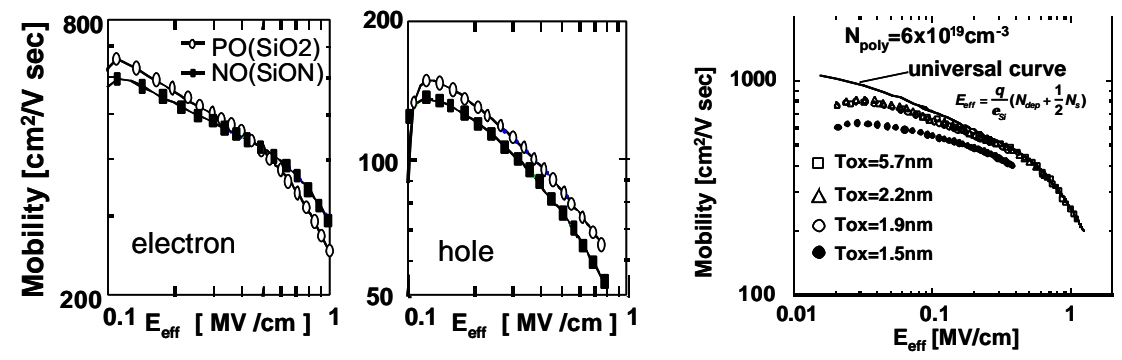

Figure 1: Dependence of effective mobility on the effective electric field, $E_{\text {eff }}$, for pure oxides and oxynitrides [1].
Figure 2: Dependence of inversion-layer mobility on effective electric field, Eeff, for nMOSFETs with different Tox [4].

The gate impurity concentration is $6 \times 10^{19} \mathrm{~cm}^{-3}$. Solid line stands for the universal mobility curve.

\section{Scattering model}

\subsection{Coulomb scattering}

As shown in Fig. 3, the MOS structure is composed of a medium with permitivity $\varepsilon_{g}$ for $\mathrm{z} \leq 0$ (gate), an insulator with permitivity $\varepsilon_{o x}$ for $0<\mathrm{z} \leq t_{o x}$ (gate oxide), and a silicon substrate with permitivity $\varepsilon_{s i}$ for $\mathrm{z}>t_{o x}$. In the MOS structure, the significant depth profile of electrostatic potential is formed as is schematically shown in Figure 3 (a). The potential distributions, $V_{\text {gate }}$ (poly-Si gate), $V_{o x}$ (gate oxide), $V_{s i}$ (substrate), are determined from the Poisson's equations in Fig. 3(a), where $\rho_{\mathrm{si}}{ }^{0}(\mathbf{R})$ and $\rho_{\text {gate }}{ }^{0}(\mathbf{R})$ are carrier distributions in the substrate and the poly-Si gate. We also assume the fixed charge distribution in the gate oxide as shown in Fig. 3.

Before starting the study, it is important to recognize that the discrete distribution of Coulomb centers is embedded in the background of the electrostatic potential with significant depth profile, as is shown schematically in Fig. 3(b). Given this standpoint, the key for the formulation of Coulomb scattering is to take into account the screening effect due to free carriers in the inversion layer [6] and in the poly-Si gate [7,8,9].

The net potential distribution $\phi(\mathbf{R})$ is determined from the general Poisson equation including the discreteness of Coulomb centers, as is shown in Fig. 3(b), 
where we express the position vector in the substrate as $\mathbf{R}=(\mathbf{r}, \mathrm{z})$ and that in the gate oxide and the poly-Si gate as $\mathbf{R}^{\prime}=\left(\mathbf{r}^{\prime}, z^{\prime}\right)$. The scattering potential $\phi_{\text {scat }}(\mathbf{R})$ responsible for Coulomb scattering needs to be extracted from $\phi(\mathbf{R})$. Here we make our first assumption, namely, that $\phi_{\text {scat }}(\mathbf{R})$ is given as

$\phi_{\text {scat }}(\mathbf{R})=\phi(\mathbf{R})-V_{j}(z)$

where j=si, ox, gate. To obtain $\phi_{\text {scat }}(\mathbf{R})$, we make our second assumption, namely, that $\rho_{\text {si }}(\mathbf{R})$ and $\rho_{\text {gate }}(\mathbf{R})$ are expanded in terms of $\phi_{\text {scat }}(\mathbf{R})$ as

$$
\begin{aligned}
& \rho_{s i}(\mathbf{R}) \cong \rho_{s i}^{0}(z)+\frac{2 \varepsilon_{s i}}{e} \sum_{i, k} s_{i}^{k} \bar{\phi}_{s c a t}^{i k}(\mathbf{r}) \\
& \rho_{\text {gate }}\left(\mathbf{R}^{\prime}\right) \cong \rho_{\text {gate }}^{0}\left(\mathbf{R}^{\prime}\right)+\frac{\varepsilon_{\text {poly }}}{e} q_{d}^{2}\left(z^{\prime}\right) \phi_{\text {scat }}\left(\mathbf{R}^{\prime}\right)
\end{aligned}
$$

where $s_{i}^{k}$ is the screening parameter for the sub-band in the valley $\mathrm{k}$ and $q_{d}^{2}\left(z^{\prime}\right)$ is the position-dependent Debye screening length which is expressed as in Fig. 4(b), and $\bar{\phi}_{\text {scat }}^{i k}(\mathbf{r}) \equiv \int d z g_{i k}(z) \phi_{\text {scat }}(\mathbf{R})$

Here $g_{i k}(z)$ is normalized charge density in the inversion layer for sub-band $i$ in valley $\mathrm{k}$.

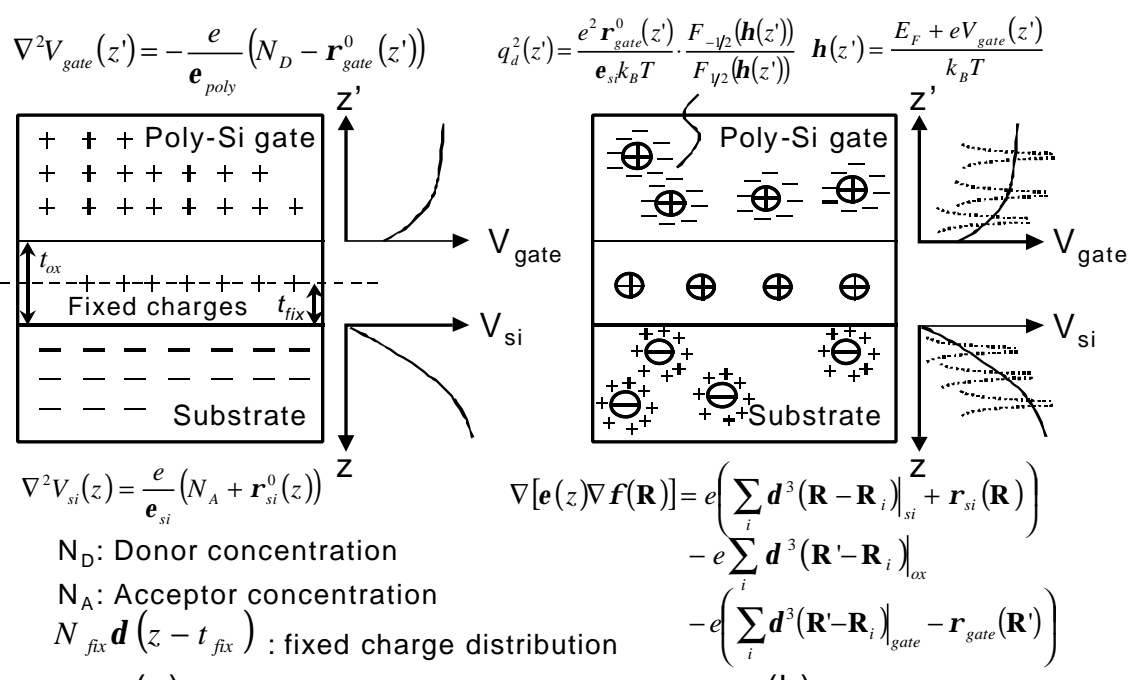

(a)

Figure 3: Schematic diagram of MOS structure.

Substituting eqs. (1), (2) and (3) into the general Poisson equation in Fig. 3(b), we obtain the Poisson equation for the net scattering potential $\phi_{\text {scat }}(\mathbf{R})$

$$
\begin{aligned}
& \nabla\left[\varepsilon(z) \nabla \phi_{\text {scat }}(\mathbf{R})\right]=-e\left(\left.\sum_{i} \delta^{3}\left(\mathbf{R}^{\prime}-\mathbf{R}_{i}\right)\right|_{g a t e}-N_{D}\right)+2 \varepsilon_{s i} \sum_{i, k} s_{i}^{k} \bar{\phi}_{s c a t}^{i k}(\mathbf{r}) \\
& -e\left(\left.\sum_{i} \delta^{3}\left(\mathbf{R}^{\prime}-\mathbf{R}_{i}\right)\right|_{o x}-N_{f i x} \delta\left(z-t_{f i x}\right)\right)+\varepsilon_{p o l y} q_{d}^{2}\left(z^{\prime}\right) \phi_{s c a t}\left(\mathbf{R}^{\prime}\right)
\end{aligned}
$$


It should be noted here that the screening effect due to free carriers in the poly-Si gate is taken into account by $\varepsilon_{p o l y} q_{d}^{2}\left(z^{\prime}\right) \phi_{\text {scat }}\left(\mathbf{R}^{\prime}\right)$ in eq. (5). Since this term vanishes except for the poly-Si gate, the screening effect due to free carriers in the poly-Si gate only acts on Coulomb scattering by impurities in the poly-Si gate (RCS). Based on eq. (5), Poisson equations for the scattering potential responsible for Coulomb scattering due to fixed charges, $\phi_{\text {scat }}^{\text {fix }}(\mathbf{R})$, and that for the scattering potential due to charged impurities in the poly-Si gate, $\phi_{\text {scat }}^{r c s}(\mathbf{R})$, are expressed as

$$
\begin{aligned}
\nabla\left[\varepsilon(z) \nabla \phi_{s c a t}^{f i x}(\mathbf{R})\right] & =-e\left(\left.\sum_{i} \delta^{3}\left(\mathbf{R}^{\prime}-\mathbf{R}_{i}\right)\right|_{o x}-N_{f i x} \delta\left(z-t_{f i x}\right)\right) \\
& +2 \varepsilon_{s i} \sum_{i, k} s_{i}^{k} \bar{\phi}_{s c a t}^{f i x, i k}(\mathbf{r}) \\
\nabla\left[\varepsilon(z) \nabla \phi_{s c a t}^{r c s}(\mathbf{R})\right] & =-e\left(\left.\sum_{i} \delta^{3}\left(\mathbf{R}^{\prime}-\mathbf{R}_{i}\right)\right|_{g a t e}-N_{D}\right)+2 \varepsilon_{s i} \sum_{i, k} s_{i}^{k} \bar{\phi}_{s c a t}^{r c s, i k}(\mathbf{r}) \\
& +\varepsilon_{p o l y} q_{d}^{2}\left(z^{\prime}\right) \phi_{\text {scat }}^{r c s}\left(\mathbf{R}^{\prime}\right)
\end{aligned}
$$

In the following, mobility limited by Coulomb scattering is calculated based on eq. (6) and (7) using relaxation time approximation. .

\subsection{Surface roughness scattering under oxynitride gate oxides}

The mobility limited by surface roughness scattering, $\mu_{S R}$, is characterized not only by $\Delta$ (roughness rms value) and $\Lambda$ (correlation length), but also by roughness power spectrum $S(q)$. Pirovano et al. [10] have proposed the functional form of

$$
S(q)=\pi(\Delta \Lambda)^{2} e^{-\frac{(q \Lambda)^{n}}{4}}
$$

The value of the parameter $n$ has been adjusted so as to successfully reproduce the universality of both electron and hole mobility under the same parameters $\Delta$ and $\Lambda$ in MOSFETs with pure oxides.

On the other hand, the use of the following roughness correlation function in real space is another possible approach to give the functional form of $S(q)$

$$
S_{r}(r)=\Delta^{2} e^{-\left(\frac{r}{\Lambda}\right)^{n}}
$$

The functional form of $S(q)$ is obtained by the Fourier transform of $S_{r}(r)$ as

$$
S(q)=2 \pi(\Delta \Lambda)^{2} \int_{0}^{\infty} d x x e^{-x^{n}} J_{0}(q \Lambda x)
$$

It has been found that hole $\mu_{S R}$ is much more sensitive to the change in $\mathrm{n}$ than electron $\mu_{S R}[11]$. This fact is the key point for the quantitative understanding of $\mu_{S R}$ in MOSFETs with oxynitride gate oxides.

In the following, $\mu_{S R}$ is calculated based on the relaxation time approximation. 


\section{Inversion-layer mobility in MOSFETs with oxynitride gate oxides}

\subsection{Low field mobility}

Fixed charges are known to exist in oxynitride gate oxides based on the observation of flat-band voltage shift, $\Delta V_{F B}$ [1]. Fixed charges cause the increase of Coulomb scattering and are considered to be the origin of the mobility degradation in low effective electric field in MOSFETs with oxynitride gate oxides [12] shown in Fig. 1.

Fig. 4 shows the calculated $N_{s}$ dependence of mobility limited by fixed charges, $\mu_{c}$, for NO oxynitride for various fixed charge densities. The value of $t_{f i x}=0.6 \mathrm{~nm}$ is used which is determined from the peak position of the Si-O-N bond measured based on Fourier Transformation InfraRed spectroscopy (FT-IR) since $\mathrm{Si}-\mathrm{O}-\mathrm{N}$ bond is considered to be the origin of the fixed charges. The good agreement between the calculation and experiment is observed in Fig. 4. It is found from Fig. 5 that the fixed charge densities extracted by the fitting method agree well with those obtained based on the flat-band voltage shift. The above results demonstrate that the mobility degradation in low effective electric field is attributable to the fixed charges.

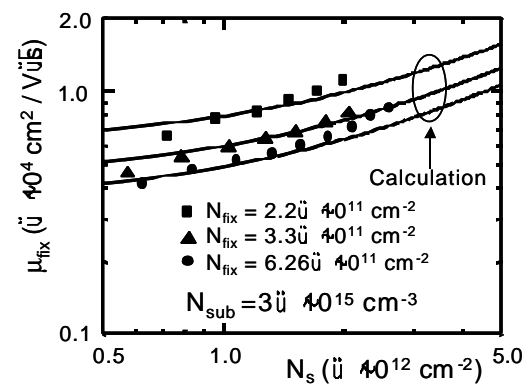

Figure 4: Ns dependence of calculated $\mu_{c}$ for NO oxynitride for various fixed charge densities [12].

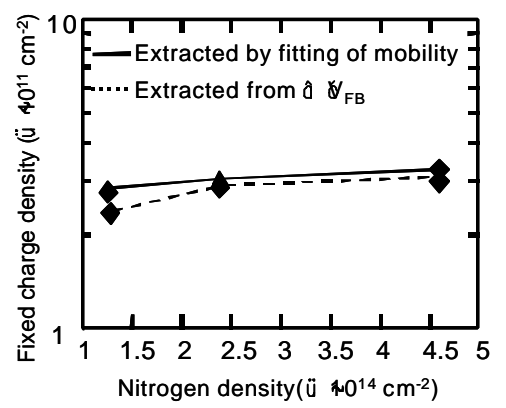

Figure 5: Comparison of the fixed charge densities obtained by $\Delta V_{F B}$ and by the fitting of $\mu_{c}[12]$.

\subsection{High field mobility}

It is found from Fig. 1 that high field mobility, limited by surface roughness scattering, $\mu_{S R}$, exhibits different behavior for electrons and holes. This incomprehensible behavior of $\mu_{S R}$ cannot be explained by the fitting of $\Delta$ or $\Lambda$. The key point for the quantitative understanding of this behavior of $\mu_{S R}$ is to find the appropriate form of the correlation function of the surface roughness, since $\mu_{S R}$ is largely affected by the change in the value of $\mathrm{n}$ through the change in the form of $S(q)$ [9].

Fig. 6 shows $S(q)$ calculated by eq. (10) in wave number space as a parameter of $\mathrm{n}$ and the range of electron and hole $k_{F}$, where Ns is varied from $5 \times 10^{12} \mathrm{~cm}^{-2}$ to $10^{13}$ $\mathrm{cm}^{-2}$; these values of Ns are typical for surface roughness scattering. Here, $k_{F}$ is Fermi 
wave number, $k_{F}=\left(2 \pi N_{s} / g\right)^{1 / 2}$, where $\mathrm{g}$ is the degeneracy in the lowest sub-band. Since $\mathrm{g}=2$ for electrons and $\mathrm{g}=1$ for holes, $k_{F}$ for holes is larger than that of electrons. Considering the fact that the inversion layer carrier gas is degenerate in the range of surface carrier concentration where $\mu_{S R}$ is dominant, $\mu_{S R}$ is mostly determined by $k_{F}$. Therefore, the behavior of $S(q)$ around $k_{F}$ is the key for the understanding of $\mu_{S R}$.

It is found in Fig. 6 that the change in the value of $n$, which determines the functional form of $S(q)$, has a much large impact on the value of $S(q)$ for holes than for electrons, because hole $k_{F}$ is larger than electron $k_{F}$. This result suggests that hole $\mu_{S R}$ is much more sensitive to the change in $n$ than electron $\mu_{S R}$. This prediction is actually confirmed in Fig. 7.

For NO oxynitride, Fig. 8 shows that when $\mathrm{n}$ is taken to be 2.0 and $\Delta$ is taken to be a smaller value $(0.45 \mathrm{~nm})$ than that with pure oxide $(\mathrm{PO})(0.55 \mathrm{~nm})$, electron and hole $\mu_{S R}$ with NO can be consistently represented under a common parameter set. The above results are schematically shown in Fig. 9. It is concluded from the analysis for the correlation function form that the increase in electron $\mu_{S R}$ with NO and the decrease in hole $\mu_{S R}$ with NO versus $\mu_{S R}$ with PO are attributable to the decrease in $\Delta$ and the increase in $\mathrm{n}$, respectively. This indicates that NO oxidation significantly changes the nature of interface geometry and suggests that the surface roughness formed by NO oxidation has more enhanced randomness (larger $\mathrm{n}$ ), while the height of roughness decreases, compared to that with thermal oxidation.

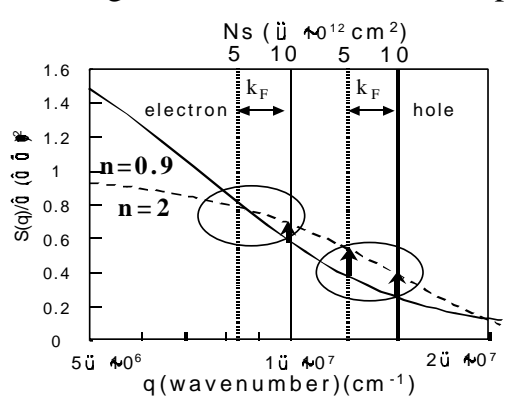

Figure 6: Power spectral densities obtained by eq. (10) as a parameter of $n$ [11].

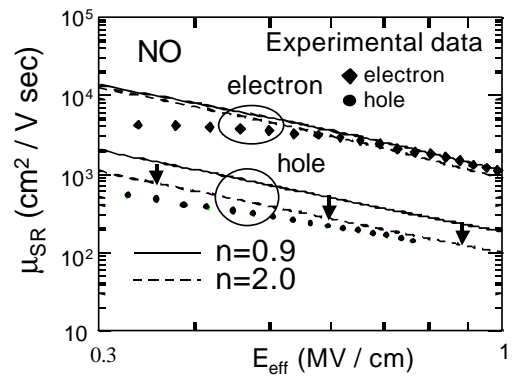

Figure 8: $E_{\text {eff }}$ dependence of $\mu_{S R}$ for NO oxynitrides. The value of $\Delta$ is taken to be $0.45 \mathrm{~nm}$ and $\Lambda$ to be $0.7 \mathrm{~nm}$ [11].

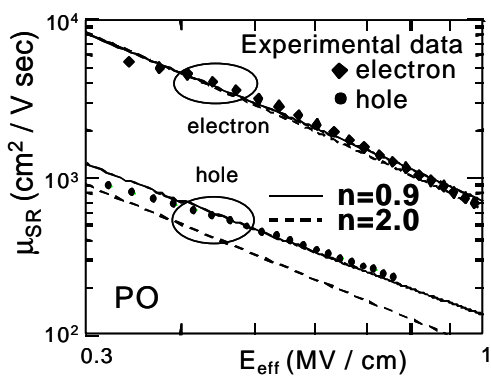

Figure 7: $E_{\text {eff }}$ dependence of $\mu_{S R}$ for PO oxides. The value of $\Delta$ is taken to be $0.55 \mathrm{~nm}$ and that of $\Lambda$ to be $0.7 \mathrm{~nm}[11]$.

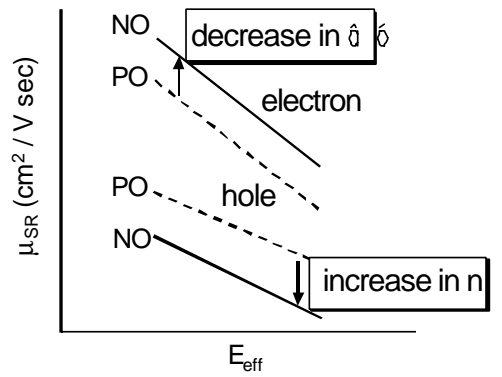

Figure 9: Schematic diagram of electron and hole of $\mu_{S R}$ for PO and NO and physical origin of the difference [11]. 


\section{Mobility degradation in MOSFETs with ultra-thin gate oxides}

It has been reported that the inversion layer mobility exhibits a significant reduction in gate oxides thinner than a critical thickness [3,4], as shown in Fig. 2. Since reliable experimental results suggest that the mobility lowering in ultra-thin gate oxides can be associated with remote Coulomb scattering (RCS) due to impurities in the poly-Si gate, we show in Fig. 10 the comparison of the experimental mobility lowering and the calculated results of $\mu_{r c s}$ by the present model described in section 2 . Note that the experimental mobility-lowering was extracted by using Matthiessen's rule. It is found that the $N_{\text {poly }}$ (impurity density in the poly-Si gate) dependence of $\mu_{r c s}$ is well represented by the present model, but not by the depletion approximation (DA) $[13,14]$. This fact indicates that (1) the experimental mobility lowering is attributable to RCS and that (2) the depletion approximation is inappropriate. As a result, the consideration of all impurities in the poly-Si gate under the screening effect due to free carriers in the poly-Si gate is essential to the description of the mobility lowering in ultra-thin gate oxides.

It is also found from Fig. 11 that the $t_{o x}$ dependence of $\mu_{r c s}$ is well reproduced by the present model. It should be noted here that $\mu_{r c s}$ in the present model provides $\exp (-$ $2 q t_{o x}$ ) dependence, where $\mathrm{q}$ is the two-dimensional wave number. The good agreement between the calculated and experimental results indicates that the mobility lowering in ultra-thin gate oxides exhibits exponential dependence on $t_{o x}$. Therefore, the mobility degradation due to RCS becomes more significant in MOSFETs with ultra-thin gate oxides.

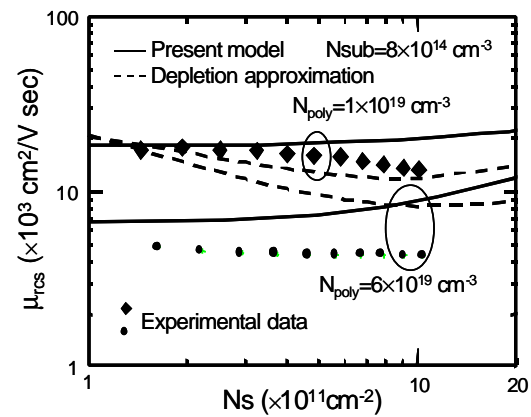

Figure 10: Comparison of the experimental mobility-lowering component [4] with $\mu_{r c s}$ calculated by the present model and by depletion approximation [7].

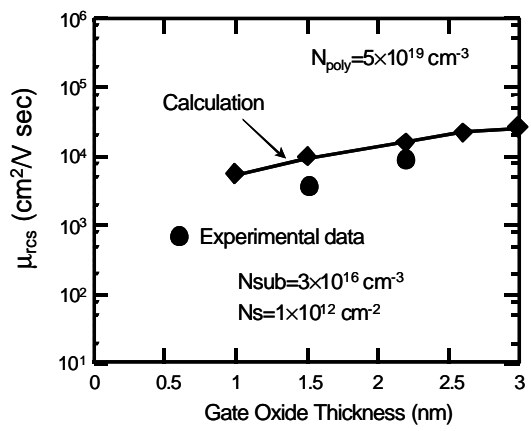

Figure 11: Comparison of the experimental mobility-lowering component [3] with $\mu_{r c s}$ calculated by the present model as a function of $t_{o x}$ at $N_{s}$ of $1 \times 10^{12} \mathrm{~cm}^{-2}[7]$.

\section{Conclusions}

Inversion layer mobility in MOSFETs with oxynitride and ultra-thin gate oxides has been quantitatively examined. For MOSFETs with oxynitride gate oxides, the 
degradation of low field mobility is attributable to the increased Coulomb scattering due to fixed charges, while the change in electron and hole mobility in high field region associated with NO oxynitridation can be reasonably well explained by the appropriate choice of the form of the roughness correlation function. For MOSFETs with ultra-thin gate oxides, a significant reduction of inversion layer mobility can be explained well by remote Coulomb scattering due to impurities in the poly-Si gate, which is formulated by taking into account the coexistence of charged impurities and free carriers in the gate region together with an appropriate consideration of the screening effect. Thus, the accurate modeling of Coulomb and roughness scattering is the key for the comprehensive understanding of carrier transport properties in MOS inversion layers.

\section{References}

[1] M. Takayanagi, and Y. Toyoshima, Tech. Dig. Int. Electron Devices Meet. (1998), pp. 575-578.

[2] T. Yamaguchi, R. Iijima, T. Ino, A. Nishiyama, H. Satake and N. Fukushima, Tech. Dig. Int. Electron Devices Meet. (2002), pp. 621-624.

[3] S. Takagi and M. Takayanagi, "Experimental evidence of inversion-layer mobility lowering in ultrathin gate oxide metal-oxide-semiconductor field-effect transistors with direct tunneling current", Jpn. J. Appl. Phys. Vol. 41, pp. 2348-2352, 2002.

[4] J. Koga, T. Ishihara, and S. Takagi, "Effect of gate impurity concentration on inversionlayer mobility in MOSFETs with ultrathin gate oxide layer", IEEE Electron Device Lett., vol. 24, pp. 354-356, 2003.

[5] S. Saito, D. Hisamoto, S. Kimura, and M. Hiratani, Tech. Dig. Int. Electron Devices Meet. (2003), pp. 797-800.

[6] F. Stern and W. E. Howard, "Properties of semiconductor surface inversion layers in the electric quantum limit", Phys. Rev. 163, pp. 816-835, 1967.

[7] T. Ishihara, J. Koga, S. Takagi, and K. Matsuzawa, Int. Conf. On Solid State Devices and Materials. (2003), pp. 12-13.

[8] D. Esseni and A. Abramo, "Modeling of Electron mobility degradation by remote Coulomb scattering in ultrathin oxide MOSFETs", IEEE Trans. Electron Devices., vol. 50, pp. 1665-1674, 2003.

[9] F. Gámiz and M. V. Fischetti, "Remote Coulomb scattering in metal-oxidesemiconductor field effect transistors: Screening by electrons in the gate", Appl. Phys. Lett., vol. 83, pp. 4848-4850, 2003.

[10] A. Pirovano, A. L. Lacaita, G. Zandler, and R. Oberhuber, "Explaining the dependence of the hole and electron mobilities in Si inversion layers", IEEE Trans. Electron Devices., vol. 47, pp. 718-447, 2000.

[11] T. Ishihara, K. Matsuzawa, M. Takayanagi, and S. Takagi, "Comprehensive understanding of electron and hole mobility limited by surface roughness scattering in pure oxides and oxynitrides based on correlation function of surface roughness", J. J. Appl. Phys., vol. 41, pp. 2353-2358, 2002.

[12] T. Ishihara, S. Takagi, and M. Kondo, "Quantitative understanding of electron mobility limited by Coulomb scattering in metal oxide semiconductor field effect transistors with $\mathrm{N}_{2} \mathrm{O}$ and NO oxynitrides", Jpn. J. Appl. Phys., vol. 40, pp. 2597-2602, 2001.

[13] N. Yang, W. K. Henson, J. R. Hauser, and J. J. Wortman, "Estimation of the effects of remote charge scattering on electron mobility of n-MOSFETs with ultrathin gate oxides", IEEE Trans. Electron Devices., vol. 47, pp. 440-447, 2000.

[14] S. Saito, K. Torii, M. Hiratani, and T. Onai, "Improved theory for remote-chargescattering-limited mobility in metal-oxide semiconductor transistors", Appl. Phys. Lett., vol. 81, pp. 2391-2393, 2002. 